\title{
A case study of low-frequency waves at the magnetopause
}

\author{
L. Rezeau ${ }^{1}$, F. Sahraoui ${ }^{2}$, E. d'Humières ${ }^{2}$, G. Belmont ${ }^{2}$, T. Chust $^{2}$, N. Cornilleau-Wehrlin ${ }^{2}$, L. Mellul ${ }^{2}$, \\ O. Alexandrova ${ }^{2}$, E. Lucek ${ }^{3}$, P. Robert ${ }^{2}$, P. Décréau ${ }^{4}$, P. Canu ${ }^{2}$, and I. Dandouras ${ }^{5}$ \\ ${ }^{1}$ CETP/UPMC, 10-12 avenue de l'Europe, 78140 Vélizy, France \\ ${ }^{2}$ CETP/UVSQ, 10-12 avenue de l'Europe, 78140 Vélizy, France \\ ${ }^{3}$ Space and Atmospheric Group, The Blackett laboratory, Imperial College, Prince Consort Rd, London, UK \\ ${ }^{4}$ LPCE, 3A avenue de la recherche scientifique, 45071 Orléans Cedex 2, France \\ ${ }^{5}$ CESR, 9 avenue du Colonel Roche, 31028 Toulouse cedex 4, France
}

Received: 2 April 2001 - Revised: 10 July 2001 - Accepted: 11 July 2001

\begin{abstract}
We present the study of one of the first magnetopause crossings observed by the four Cluster spacecraft simultaneously, on 10 December 2000. Although the delays between the crossings are very short, the features of the boundary appear quite different as seen by the different spacecraft, strongly suggesting the presence of a local curvature of the magnetopause at that time. The small-scale fluctuations observed by the STAFF search-coil experiment are placed in relation to this context. A preliminary investigation of their behaviour on the boundary and in the neighbourhood magnetosheath is performed in comparison with the theoretical model of Belmont and Rezeau (2001), which describes the interaction of waves with the boundary.
\end{abstract}

Key words. Space plasma physics (transport processes, discontinuities, turbulence)

\section{Introduction}

For many years, scientists have tried to understand the transfer of particles through the magnetopause. Various models have been used with this aim in mind with the main difficulty arising from the fact that the plasma around the magnetopause is collisionless and that usual diffusion cannot be invoked. Different experimental studies had given the indication that the small-scale electromagnetic fluctuations are likely to play a significant role in these transfers, by taking the place of the collisions, in particular, the observation of a high level of fluctuations right at the magnetopause. Most of the models, until recently, were based on the assumed existence of local instabilities at the boundary, giving rise to plasma penetration, through either anomalous diffusion or reconnection (tearing instability). Belmont and Rezeau (2001) have recently proposed a different and probably more realistic model for explaining both the origin of the strong magnetopause fluctuations and the mechanism of transfer.

Correspondence to: L. Rezeau

(laurence.Rezeau@cetp.ipsl.fr)
These authors suggest that the primary cause lies not in a local instability, but in the pre-existing magnetosheath fluctuations; by studying the propagation of incident magnetosheath waves through the magnetopause, they have shown that these waves convert into Alfvén waves in the boundary density gradient. Moreover, in the presence of a sufficient magnetic field rotation, the resulting Alfvén waves are shown to be trapped in the boundary, therefore producing a local enhancement of the fluctuation level. The major consequence of this trapped small-scale turbulence is to allow via Hall-MHD effects a micro-reconnection distributed all over the boundary.

The time has now come to confront the model with the Cluster data. Part of the Wave Experiment Consortium (Pedersen et al., 1997), namely the STAFF instrument, is devoted to the measurement of the electromagnetic fluctuations ranging from 0 to $4 \mathrm{kHz}$ (Cornilleau-Wehrlin et al., 1997). The STAFF-SC part of the instrument gives the waveform of the low-frequency range (up to $10 \mathrm{~Hz}$ ) magnetic fluctuations. These data are used primarily in the present analysis, together with data that allow for the large-scale description of the magnetopause: the Flux-Gate Magnetometer (FGM) data (Balogh et al., 1997), the density data from the WHISPER instrument (Décréau et al., 1997), and the particle measurements made by CIS (Rème et al., 1997).

The two magnetopause crossings that are studied here have been chosen only because they were observed during one of the first periods when data were acquired simultaneously on the four spacecraft. For both crossings, the magnetic field rotates more than $90^{\circ}$, which is favourable for the scenario to be tested. Such large-scale features of the boundary are presented in Sect. 2, using FGM data, in order to replace the electromagnetic fluctuations in their context. Section 3 is devoted to the study of the magnetic fluctuations themselves. To investigate the modes observed around the boundary, a minimum variance analysis is performed on the STAFF data. These results are very preliminary, since no other case has been studied yet and no specific multispacecraft tool has been used yet for the study. 
Table 1. First crossing: Comparison of the directions of the magnetopause normal determined by Sibeck's model, the CP, and the MVA methods, respectively

\begin{tabular}{cccc}
\hline $\begin{array}{c}\text { Angle between } \\
\text { S/C }\end{array}$ & CP and model & MVA and model & CP and MVA \\
\hline 1 & $23.2^{\circ}$ & $40.2^{\circ}$ & $17.4^{\circ}$ \\
2 & $21.5^{\circ}$ & $31.2^{\circ}$ & $12.1^{\circ}$ \\
3 & $21.0^{\circ}$ & $43.5^{\circ}$ & $22.7^{\circ}$ \\
4 & $19.1^{\circ}$ & $39.9^{\circ}$ & $21.0^{\circ}$ \\
\hline
\end{tabular}

\section{Large-scale behaviour of the magnetopause}

On 10 December 2000, the WIND satellite is not in a very good position to place our study in its interplanetary context (GSM: $28.5 R_{E}, 185.0 R_{E},-50.5 R_{E}$ ). However, it indicates that the pressure of the solar wind and its speed are rather steady around $2 \mathrm{nPa}$ and $700 \mathrm{~km} \mathrm{~s}^{-1}$, respectively. $B_{Z}$ reverses at just around 8:30, going from $5 \mathrm{nT}$ to $1 \mathrm{nT}$, ti.e. after the crossings studied here.

Since the magnetopause is, by definition, a magnetic boundary, the identification of the crossings is made first on the magnetic field data. Nevertheless, the previous studies of these regions have shown that the behaviour of the lowfrequency waves (ULF-ELF) changes radically at the crossing: the level of turbulence is low in the magnetosphere, high in the magnetosheath and even higher right on the magnetopause (Perraut et al., 1979; Anderson et al., 1982; Rezeau et al., 1989); this gives another means of identifying the magnetopause. On 10 December 2000, the orbit of the spacecraft is outbound and many magnetopause crossings can be identified in quite a short period (Fig. 1), indicating that the magnetopause is not stationary.

To make a detailed study, we select two successive crossings: around 08:15, the spacecraft are in the magnetosheath; they cross the magnetopause around 08:21 to enter the magnetosphere and then go back to the magnetosheath around 08:25:50. At this time, the spacecraft explore the boundary of the magnetosphere on the evening side, well above the ecliptic plane $\left(X_{\mathrm{GSE}}=0.3 R_{E}, Y_{\mathrm{GSE}}=18 R_{E}, Z_{\mathrm{GSE}}=6 R_{E}\right)$. As learned from previous one-spacecraft experiments, multiple boundary crossings can be the signatures of either a global back and forth motion of the magnetopause, or surface wave oscillations on the boundary (see for instance, Aubry et al., 1971). Using the Cluster facility, we should be able to disentangle these interpretations.

To study the large-scale behaviour of the magnetopause, we have used the FGM data with a $4 \mathrm{~s}$ resolution, together with the WHISPER data, which give an indication of the variations in the density during the crossings. These data are used first to locate the spacecraft in the different regions; and afterwards to characterize the shape of the magnetopause during both crossings. We assume the limits of the magnetopause are the times when the magnetic field stops rotating. As can be seen in Fig. 2, the two crossings look quite differ-
Table 2. Same as Table 1 for the second crossing

\begin{tabular}{cccc}
\hline $\begin{array}{c}\text { Angle between } \\
\text { S/C }\end{array}$ & CP and model & MVA and model & CP and MVA \\
\hline 1 & $73.1^{\circ}$ & $78.1^{\circ}$ & $9.1^{\circ}$ \\
2 & $51.6^{\circ}$ & $76.4^{\circ}$ & $53.7^{\circ}$ \\
3 & $84.3^{\circ}$ & $72.2^{\circ}$ & $15.0^{\circ}$ \\
4 & $81.5^{\circ}$ & $74.1^{\circ}$ & $11.6^{\circ}$ \\
\hline
\end{tabular}

ent, but both seem to be the superposition of two phenomena: a smooth variation of the modulus of the magnetic field and of the density (shown by a blue shading), and sharp rotations (shown by a pink shading). A boundary layer can be seen on the magnetospheric side of the magnetopause in the WHISPER data.

Without performing a very detailed study, some information can be obtained from the variations in the magnetic field. We used two different methods to study the magnetopause normal. First, we used the simplest approach, which consists of performing the cross product $(\mathrm{CP})$ of the averaged magnetic fields on both sides. Second, we performed a Minimum Variance Analysis (MVA) (Sonnerüp and Scheible, 1998). Both results are then compared to a model normal using Sibeck's model (parabolic shape adjusted to the position where the magnetopause is observed, as calculated by Sibeck et al., 1991), and summarized in Tables 1 and 2.

The CP method is valid whenever one can assume that the boundary is approximately tangential and that the total rotation is not too close to $0^{\circ}$ or $180^{\circ}$. The comparison between the normals obtained by this method and the normal deduced from the model is presented in the first columns of Tables 1 and 2. One observes that for both crossings, they are always different from each other. During the first crossing, the magnetic field rotates by about $125^{\circ}$ on the four spacecraft. The angle between the observed normal and the model one is about $22^{\circ}$, with the observations on the four spacecraft being similar. Furthermore, the normals of the first crossing are very different from those of the second crossing. During the second crossing, the normals are also different from satellite to satellite. Three of the $\mathrm{CP}$ normals are about $80^{\circ}$ from the model normal, while the fourth one is at $\approx 50^{\circ}$. The four normals are not in the same plane, which seems to indicate a fully three-dimensional structure of the boundary. Nevertheless, these determinations are likely to involve large uncertainties since the rotation angle is not far from $180^{\circ}$ (from $164^{\circ}$ to $172^{\circ}$, depending on the spacecraft), and a more detailed study of this case is therefore necessary.

The MVA method is actually an efficient one as long as the magnetic field variations are polarized in a plane and not along one unique direction; the validity of the result under these conditions is insensitive to the particular case of a $180^{\circ}$ rotation and gives a more reliable determination of the mag- 


\section{December 2000 STAFF}

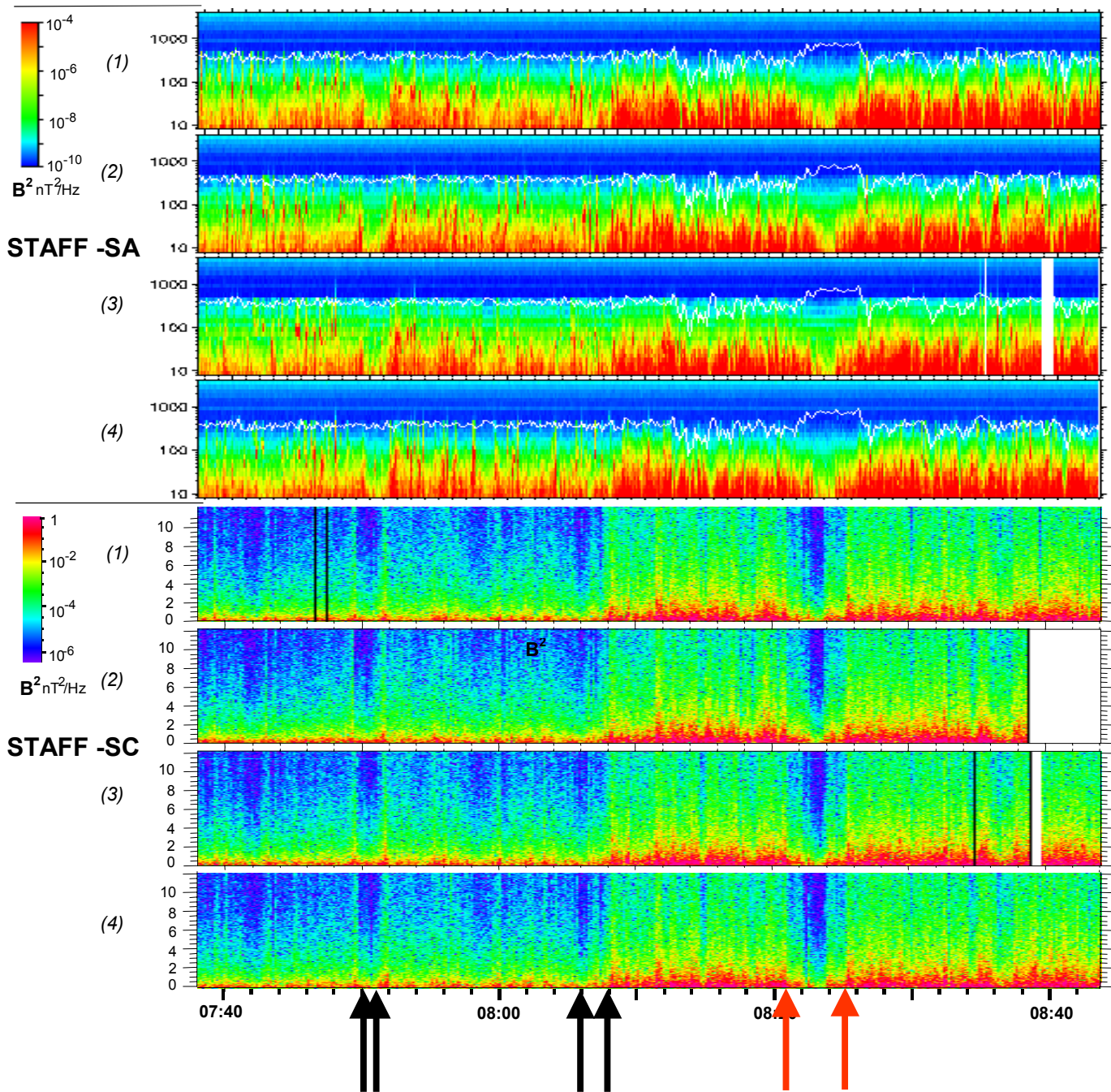

Fig. 1. Colour spectrogram of the STAFF data (the horizontal scale is time, the vertical scale is frequency in Hz). The four lowest panels show the STAFF-SC part, below $10 \mathrm{~Hz}$. The four top panels show the STAFF-SA high frequency band $(10 \mathrm{~Hz}-4 \mathrm{kHz})$. The thin white line superimposed on the spectrograms is the electron gyro-frequency as deduced from FGM magnetic field. Many magnetopause crossings can be identified: they are identified by arrows on the plot. Red arrows show the two crossings studied in this paper.

netopause normals. The first crossing lasts almost $30 \mathrm{~s}$. We used different time lengths for the minimum variance analysis, ranging from 20 to $110 \mathrm{~s}$. The results are quite stable and are not very different from the previous analysis using only the cross product: we have a mean change of $16^{\circ}$ (see columns 2 and 3 of Table 1). For the second crossing, which lasts for $20 \mathrm{~s}$, we used times ranging from $15 \mathrm{~s}$ to 1 or $2 \mathrm{~min}$. The results are also quite stable. As seen in Table 2, the main difference with the cross product analysis concerns spacecraft 2, whose normal comes close to the common value of the other spacecraft (about $76^{\circ}$ from the model normal), instead of being singular. In the actual conditions of almost $180^{\circ}$ rotations, it is clear that the MVA results are the most reliable, and we will keep them for reference in the following analyses (Sect. 3). All the preceding study tends to show that we are not observing a global back and forth motion of the magnetopause, but more likely a corrugated surface. The fact that the magnetopause behaves as a surface wave is very important for the study of the small-scale electromagnetic fluctuations on the boundary. As a matter of fact, a previous study performed on ISEE data (Rezeau et al., 1992) has shown that the power of the fluctuations was strongly dependent on the large-scale characteristics of the magnetopause, showing (i) that the power of the fluctuations is higher when the boundary is moving earthward and the magnetosphere is compressed, than when it is moving sunward, (ii) when an oscillation is observed on the boundary, the power seemed to be higher on the leading edge of the wave. This study was preliminary and not comprehensive since the measurements were performed with only two spacecraft. Cluster will provide a better view of this problem.

Some additional information on the motion of the bound- 
10 December 2000 FGM \& WHISPER
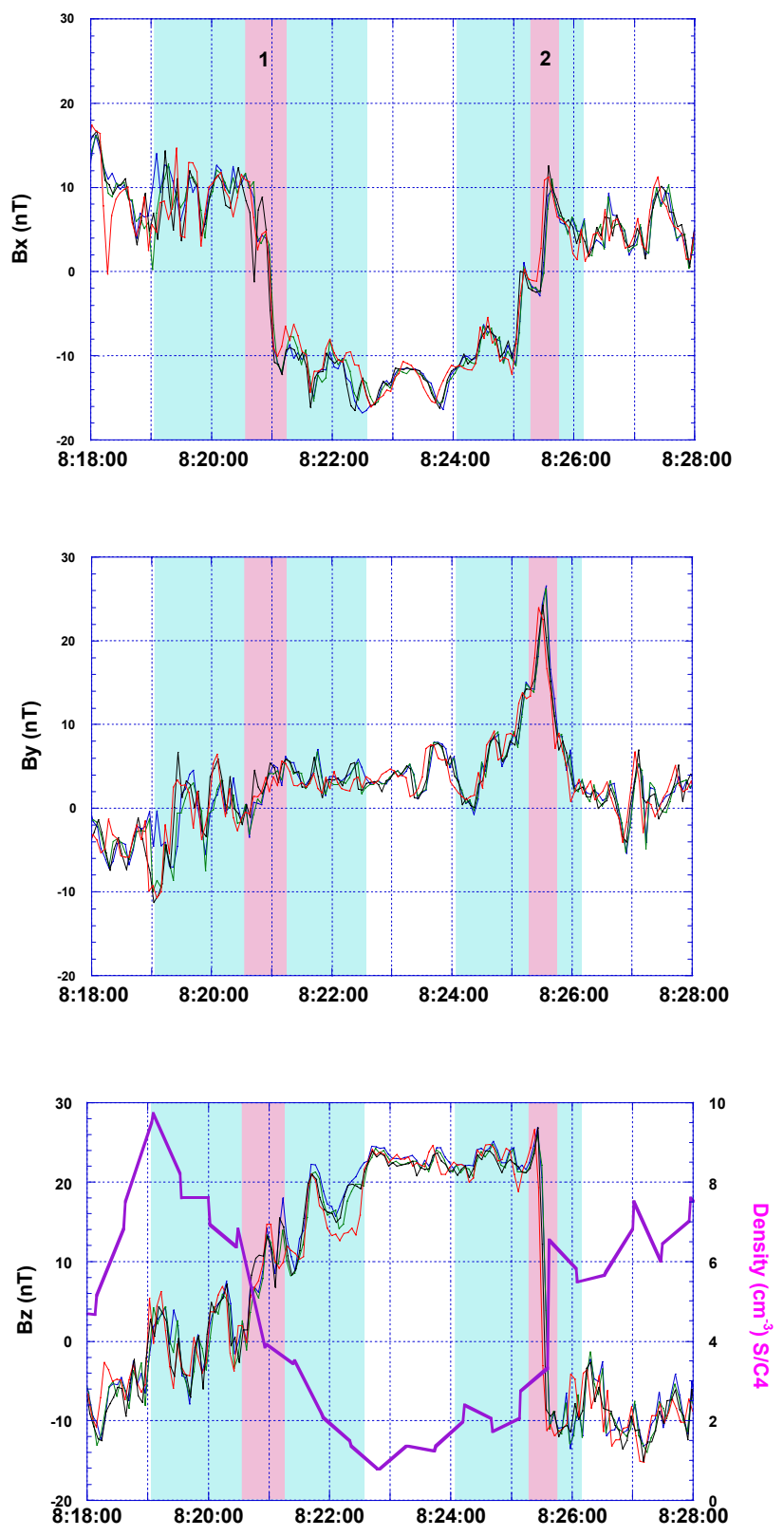

Fig. 2. Three components of the FGM magnetic field projected in the GSE frame. The time resolution is $4 \mathrm{~s}$. Each spacecraft is identified by the referenced colours (1, black, 2, red, 3, green, 4, blue). In the third panel, an estimate of the density deduced from the WHISPER experiment is superimposed. Blue shading corresponds to the density and magnetic field modulus variations. Pink shading corresponds to magnetic field rotations.

ary can be obtained from the analysis of the delays between the crossings by the different spacecraft. The method that we used for this first estimation is a simple one: for each pair of spacecraft, we assume that the magnetopause is locally a plane moving in the direction of a unique normal $\boldsymbol{n}$. The velocity of the boundary is determined by $V_{12}=\boldsymbol{d}_{12} \cdot \boldsymbol{n} /\left(t_{2}-t_{1}\right)$, where $\boldsymbol{d}_{12}$ is the vector joining the two spacecraft and $t_{2}-t_{1}$ is the delay between the two crossing signatures. As the tetrahedron is not very large (the maximum distance between spacecraft is $1000 \mathrm{~km}$ ), the estimation accuracy has to be checked carefully. A reliable value for the delay can be obtained only when the signatures obtained on the two considered spacecraft are sufficiently similar. The parameter that has been used to determine the crossings is the angle between the magnetic field and a fixed direction in the tangential plane. It gives a clear signature of the rotation of the magnetic field. With this parameter, both crossings display a relatively simple structure. For the second crossing, the signatures appear very similar on spacecraft 2 and 4, and fairly similar on spacecraft 1 , but spacecraft 3 displays a very different signature, which remains to be explained. Using the spacecraft 2 and 4 , a delay of $\approx 4.8 \mathrm{~s}$ is observed, leading to a magnetopause velocity of about $230 \mathrm{~km} \mathrm{~s}^{-1}$ (along the local normal). For the first crossing, the delays between the spacecraft are shorter $(\approx 1 \mathrm{~s})$, and the accuracy of the velocity determination is less reliable. From spacecraft 3 and 4, one obtains nevertheless, a magnetopause velocity of about $154 \mathrm{~km} \mathrm{~s}^{-1}$.

It is quite interesting to compare the magnetopause velocities obtained in this way with the ion velocities measured on board Cluster. Even if the normal component $B_{n}$ has been found to be significantly different from zero, which indicates that the magnetopause is not strictly tangential and that the magnetosheath and magnetosphere magnetic field lines are "connected", it is to be expected that the difference between the normal components of these two velocities remains small. We tried to check this with CIS experiment, using CODIF data, which are available on spacecraft 4 , and HIA data, which are only available on spacecraft 3 . CODIF count rates are partially saturated in the magnetosheath, which results in underestimated velocity values; however, the trends in the velocity direction variations are the same as on HIA. When looking at $3 \mathrm{mn}$ averaged values in the adjacent magnetosheath or magnetosphere, the ion velocity appears almost tangential to the model magnetopause, independent of the corrugation. When looking at the instantaneous values in the magnetosheath closer to the boundary, the flow appears to turn rather suddenly in the limit of the $4 \mathrm{~s}$ resolution, and the velocity along the local normal seems to be close to the velocity of the boundary $\left(250 \mathrm{~km} \mathrm{~s}^{-1}\right.$ on CODIF, $350 \mathrm{~km} \mathrm{~s}^{-1}$ on HIA). These results are in accordance with the physical guess, but are still preliminary.

The values obtained for the magnetopause velocities of about $150-250 \mathrm{~km} \mathrm{~s}^{-1}$ may appear rather high, but it is worth noticing that such high values of the normal magnetopause velocity have already been pointed out in the literature using the two spacecraft INTERBALL investigations (Safrankova et al., 1997). All the preceding results intend to place the wave observations in their large-scale context. Figure 3 summarizes the main characteristics relevant for this goal. The integrated wave amplitude is placed on a plot where the variations in the Alfvén velocity are displayed (from CIS and FGM data), as well as the magnetic field rotation angle. The Alfvén velocity gives a clear signature of the boundary layer 
10 December 2000

B FGM rotation in the L,M plane satellite 3

STAFF integrated power $\left(\times 25 \mathrm{nT}^{2}\right)$

Alfven speed (x2000 km/s)

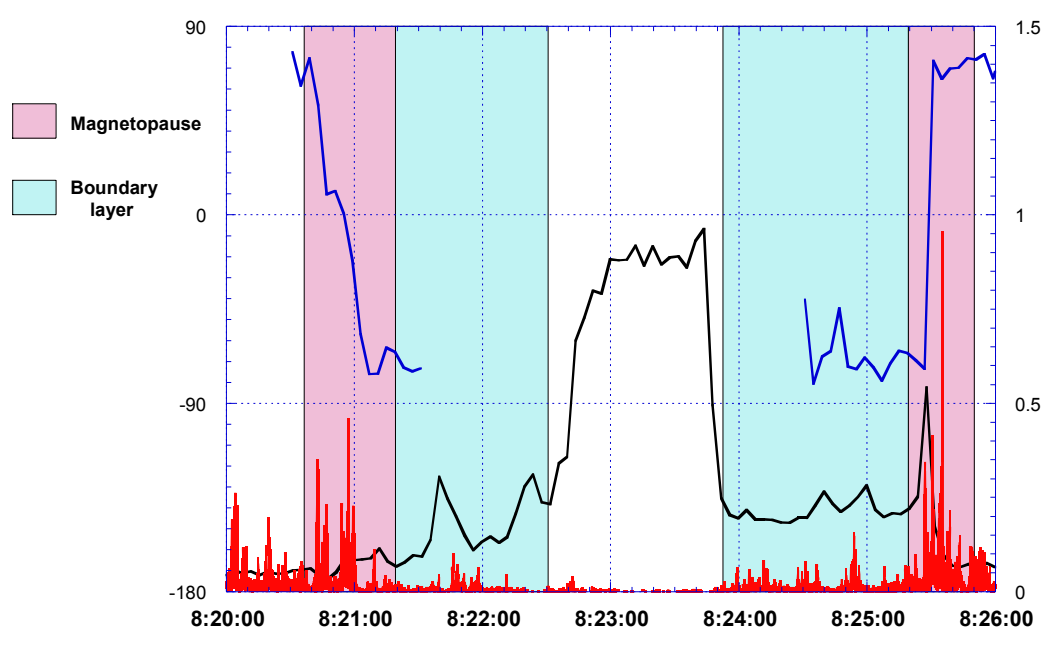

Fig. 3. Main characteristics of the waves at the magnetopause on spacecraft 3: the Alfvén speed calculated using the density is given by CIS, the magnetic field is given by FGM, the power of the fluctuations from the STAFF experiment (integrated between 0.1 and $10 \mathrm{~Hz}$ ) are normalised to 1 , and the magnetic field rotation angle is the angle between $\boldsymbol{B}_{0}$ and the $M$ direction in the $(L, M)$ plane. The angle is interrupted in the magnetosphere because the $(L, M)$ plane is determined for each crossing and there is no relation between both calculations.

Fig. 4. Power of the fluctuations from the STAFF experiment on spacecraft 2, integrated between 0.1 and $10 \mathrm{~Hz}$. and shows a local maximum in the second crossing.

\section{Small-scale fluctuations}

The first information concerning the small-scale variations comes from the distribution of power in the different regions (Fig. 4). As previously observed, the maximum of the power is seen very close to the magnetopause gradient and the level seen in the neighbouring magnetosheath is much lower. The boundary layer corresponds to a level that is comparable to the magnetosheath one. Nevertheless, the contrast between magnetosheath and magnetopause levels appears in this case, weaker than previously reported. Before the first crossing, in particular, when the spacecraft are in the magnetosheath, a very high turbulence is present: it can be seen both at large- scales on the FGM data and at smaller-scales in STAFF data. This observation might be explained by the fact that Cluster crosses the magnetopause on the flanks of the magnetosphere and at a high latitude, which is quite different from previous studies.

The study of the spectra of the fluctuations at the magnetopause can be expected to yield precious clues for understanding the behaviour of the turbulence near the magnetopause. These spectra are found in this case to follow a $f^{-\alpha}$ power law (Fig. 5), as already observed (Rezeau et al., 1999). A calculation of the parameter $\alpha$ in the magnetosheath and at the magnetopause gives values between 2.3 and 2.9, depending on the spacecraft and on the interval studied. These values are consistent with those usually observed except that, contrary to the previous cases, no systematic difference can be evidenced between the slopes at the magnetopause and 


\section{December 2000 STAFF satellite 3}

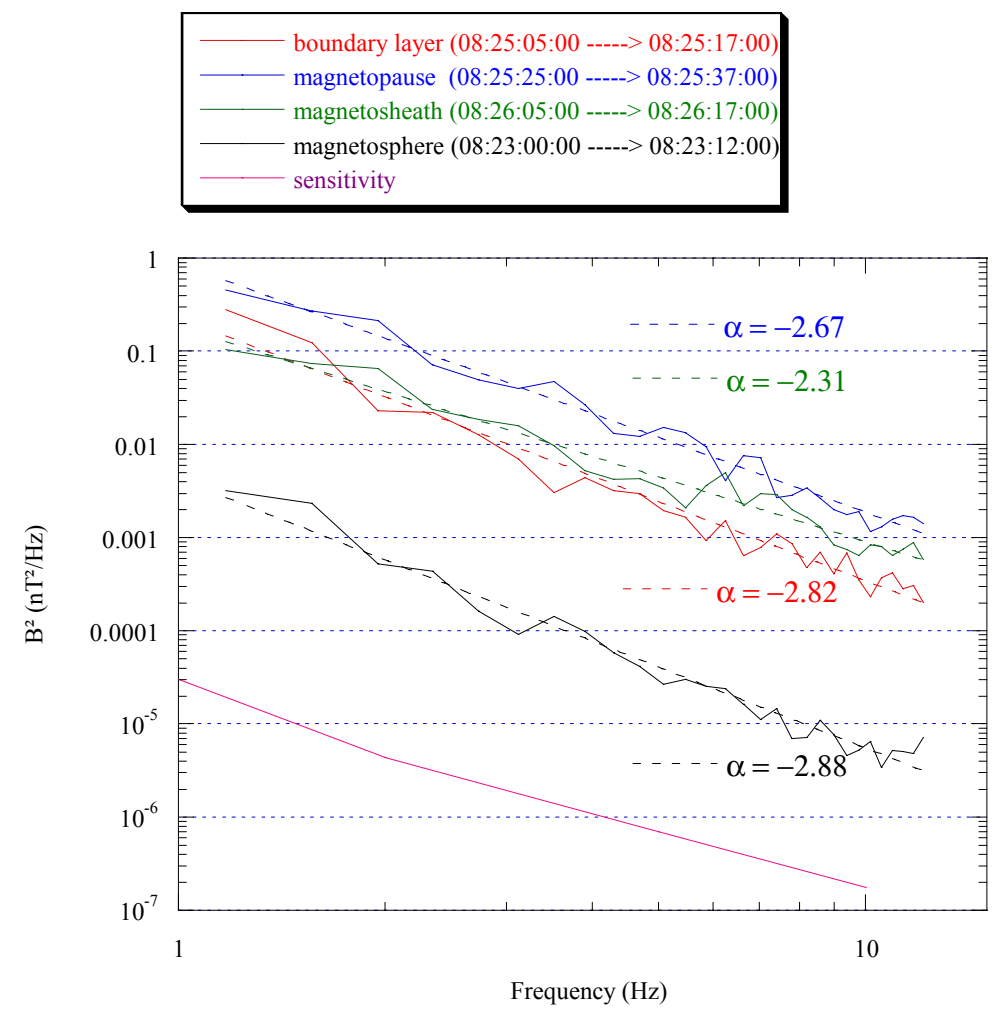

Fig. 5. Comparison of the spectra in the regions adjacent to the magnetopause on spacecraft 2. For reference, the sensitivity of the instrument is plotted showing that the fluctuations are much higher, even in the magnetosphere.

in the magnetosheath. This may reinforce the observation made on the integrated power: there is less difference between the magnetopause and the adjacent regions observed at high latitudes on the flanks of the magnetosphere than in regions closer to the front of the magnetosphere and at low latitudes.

To test the Belmont and Rezeau (2001) model, it is necessary to know how the fluctuations are polarized and whether their polarizations change across the magnetopause. This study has been performed using again a MVA method. The results presented afterward have been obtained for crossing 2 , with data filtered in a narrow frequency range $(\approx 0.1 \mathrm{~Hz})$ around $0.35 \mathrm{~Hz}$. This frequency is below the proton gyrofrequency in the magnetosphere $(\approx 0.4 \mathrm{~Hz})$ and above the magnetosheath one $(\approx 0.15 \mathrm{~Hz})$. Under the assumption that each data set analyzed is the signature of a plane wave, i.e. with a unique wave vector direction, the MVA technique can indeed provide the main properties of this plane wave: the propagation direction is the direction of minimum variance, and the polarization, linear or elliptic (in the plane perpendicular to propagation), is derived from the maximum and intermediate variances. In the present data, the ratios $\sigma_{3} / \sigma_{1}$ of minimum over maximum variances are found, on average, to be as small as 0.05 . The smallness of this ratio evidences the good planarity of the magnetic hodograms that can be drawn as functions of the spacecraft time. In a first step, we used data filtered with a high-pass fil- ter at $0.35 \mathrm{~Hz}$, but without filtering the high frequencies. The minimum over maximum variance ratios were then about 0.27 , indicating that under these conditions, the magnetic variations are not embedded in a plane but in a volume that is still greatly flattened in one direction.

The results obtained in this way have been compared to the main characteristic large-scale directions in the magnetopause vicinity. Figure 6 displays the maximum and minimum variance directions as functions of time with respect to the magnetic field direction. To obtain these results, a MVA has been performed on successive $3 \mathrm{~s}$ intervals of STAFF data centred around each data point of FGM, i.e. every $4 \mathrm{~s}$. When comparing the mean angles obtained in the magnetosphere and in the magnetosheath, one can see that there exists a significant trend. The maximum variance is rather perpendicular to $\boldsymbol{B}_{0}$ in the magnetosphere (mean value close to $70^{\circ}$ ), indicative of an "Alfvén type" polarization, while the compressional component, parallel to $\boldsymbol{B}_{0}$, is much larger in the magnetosheath (mean value close to $40^{\circ}$ ). The lowest value lies at the crossing itself where the maximum variance is at only $30^{\circ}$ from $\boldsymbol{B}_{0}$.

The angle between the minimum variance direction (supposedly the wave vector direction) and $\boldsymbol{B}_{0}$ follows an anticorrelated variation, i.e. rather parallel in the magnetosphere and more perpendicular in the magnetosheath, with a maximum close to $80^{\circ}$ at the magnetopause itself. The error bars are generally very large, suggesting that the polariza- 


\section{December 2000}

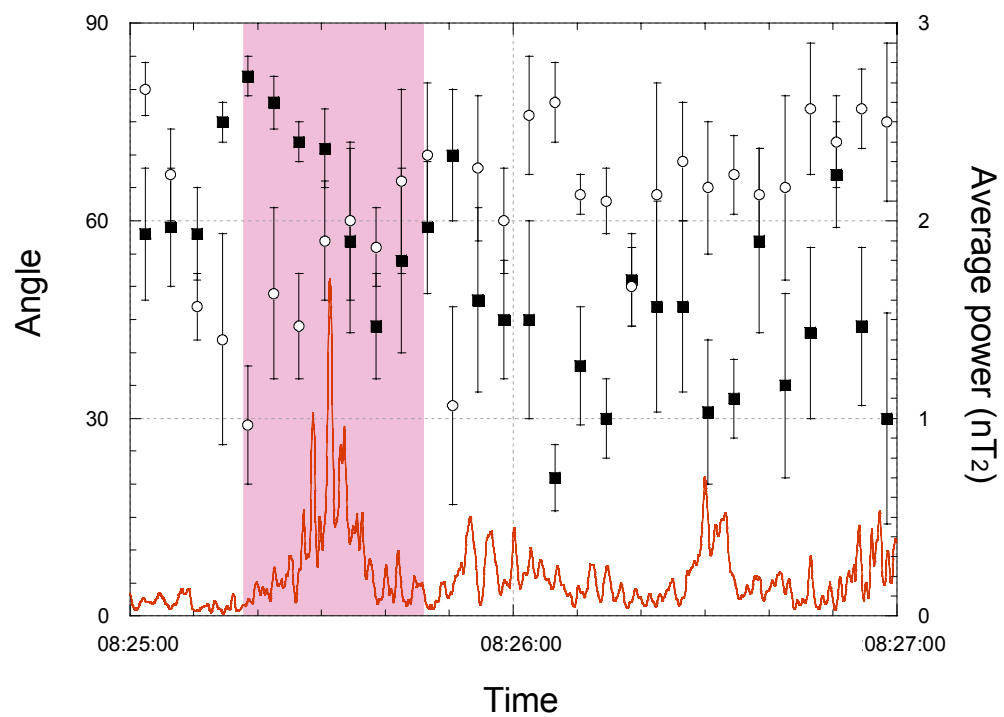

Fig. 6. MVA results for $0.35 \mathrm{~Hz}$ fluctuations for crossing 2. The points are the values of the angles between $\boldsymbol{B}_{0}$ and the direction of maximum variance (-) and minimum variances $(0)$ averaged on the four spacecraft. The error bars are the mean square differences of the four values with this average. The position of the magnetopause is shown by the shaded zone and the average power of the fluctuations is drawn for comparison. tion directions have a great random component from place to place and therefore, that the waves observed are not planar at the scale of the Cluster tetrahedron. It must be kept in mind that the planarity of the magnetic hodogram as a function of time is not, in general, a proof that we are actually dealing with plane waves. The planarity of the wave surfaces concerns the spatial and not temporal variations of the magnetic field; a plane hodogram can be considered as an indication of plane waves only when its planarity cannot be attributed to temporal variations that are too simple (in particular, monochromatic). This is probably not the case for the filtered data. This problem will not be solved without using a true 4-spacecraft method, such as multi-spacecraft filtering (Pinçon and Lefeuvre, 1998). This work is in progress.

The error bars obtained with the MVA technique are minimum close to the magnetopause crossing, rather on the magnetospheric side of the boundary. The maximum amplitude peak is clearly right in the boundary. The small error bars indicate that at this point one obtains reliable directional indicators of the strong waves: a rather parallel propagation, and a shear Alfvén type polarization. A careful comparison with the model remains to be done.

A comparison has also been performed between the maximum and minimum variance directions and the magnetopause normal. The main result is that at the point of maximum amplitude, where the uncertainties are the lowest, the minimum variance direction is almost aligned with the magnetopause normal. This is in agreement with the fact that the wave vector along the normal direction could increase in this region to values much greater than the tangential components, as found in the model of Belmont and Rezeau (2001). Outside the magnetopause, no clear trend is observed.

The same work has been done at a frequency around $2 \mathrm{~Hz}$, which is larger than the ion gyrofrequency on both sides of the magnetopause. The results appear to be rather similar on the magnetospheric side, but a clear trend not longer appears any more in the magnetosheath or at the magnetopause.

\section{Conclusion}

The results presented here are very preliminary and they need to be confirmed by further studies. They already give a glimpse of the richness of the Cluster data. Looking at a boundary such as the magnetopause with one spacecraft gives a rather simple image. Later on, by using two spacecraft missions such as ISEE 1 and 2 (Berchem and Russell, 1982) and INTERBALL-1/MAGION4 (Safrankova et al., 1997) a moving and oscillating layer was detected. Using now the Cluster "microscope" clearly increases the complexity of the interpretation. First of all, the large-scale structure of the boundary shows that the layer is not a plane at the scale of the tetrahedron, i.e. some $1000 \mathrm{~km}$, which is very small with respect to the scale of the magnetosphere. It probably cannot be approximated by a two-dimensional surface; rather, it is likely to be fully 3-dimensional locally, which is somewhat far from most models that have described it in the past. From a theoretical point of view, this observation could be related to the Kelvin-Helmholtz instability, whose growth rate is known to maximise for wave numbers of the order of the inverse thickness of the boundary (see, for instance Miura and Pritchett, 1982; Belmont and Chanteur, 1989). For the sake of simplicity, if one visualises the magnetopause as a layer of thickness $d$ limited by two discontinuities, then the theoretical linear results show that: (i) for $k d \ll 1$, the growth rate increases linearly with $k$, and the undulations on the two edges are in phase (in this condition, the infinitely thin layer approximation is correct); (ii) for increasing $k d$, the growth rate increases more slowly where the role of the 
thickness is to introduce an increasing phase difference between the two edges; (iii) for $k d \approx 1$, the growth rate stops increasing, with the phase difference reaching $180^{\circ}$; (iv) for $k d>1$, the growth rate falls rapidly down to zero. In consequence, as long as the linear fastest growing mode is the most commonly observed, one cannot expect to observe a boundary that would be locally plane. The interpretation of the magnetopause corrugations due to solar wind pressure pulses can lead to similar conclusions.

The wave observations made here confirm the main results obtained by previous experiments. In particular, the observation of a high turbulence level at the magnetopause reinforces the idea that the magnetic fluctuations play a significant role in the physics of this boundary. Nevertheless, most of the previous statistical studies of the turbulence have been performed in the front region of the magnetopause. The example shown here is quite different, since it is an observation of the magnetopause on the flanks of the magnetosphere and at high latitudes. The interaction between the magnetosheath turbulence and the boundary might be different in this region due to, for instance, a large plasma flow. This should be investigated using plasma data in the near future.

As a result of the study performed on ISEE data (Rezeau et al., 1992), the role of a surface wave on the boundary is important. Therefore the identification and the interpretation of such oscillations are of primary importance for the understanding of small-scale fluctuations.

Acknowledgements. The STAFF experiments realisation and data analysis have been supported by ESA and CNES grants. The authors wish to thank the STAFF-SA team for providing the data shown on Fig. 1.

The Editor in Chief thanks two referees for their help in evaluating this paper.

\section{References}

Anderson, R. R., Harvey, C. C., Hoppe, M. M., Tsurutani, B. T., Eastman, T. E., and Etcheto, J.: Plasma Waves Near the Magnetopause, J. Geophys. Res., 87, 2087-2107, 1982.

Aubry, M. P., Kivelson, M. G., and Russell, C. T.: Motion and structure of the magnetopause, J. Geophys. Res., 76, 1673-1696, 1971.

Balogh, A., Dunlop, M. W., Cowley, S. W. H., et al.: The Cluster Magnetic field Investigation, Space Science Reviews, 79, 1/2, 107-136, 1997.

Belmont, G. and Rezeau, L.: Magnetopause reconnection induced by magnetosheath Hall-MHD fluctuations, to appear in J. Geo- phys. Research, 2001.

Belmont, G. and Chanteur, G.: Advances in magnetopause KelvinHelmholtz instability studies, Phys. Scripta, 40, 124-128, 1989.

Berchem, J. and Russell, C. T.: Magnetic field rotation through the magnetopause: ISEE 1 and 2 Observations, J. Geophys. Res., 87, 8139-8148, 1982.

Cornilleau-Wehrlin, N., et al.: The CLUSTER Spatio-Temporal Analysis of Field Fluctuations (STAFF) Experiment, Space Science Reviews, 79, 1/2, 107-136, 1997.

Décréau, P. M. E., et al.: WHISPER, A resonance sounder and wave analyser: performances and perspectives for the Cluster mission, Space Science Reviews, 79, 1/2, 107-136, 1997.

Dunlop, M. W. and Woodward, T. I.: Multispacecraft discontinuity analysis: orientation and motion, in: Analysis Methods for multispacecraft data, (Eds) Paschmann, G. and Daly, P. W., ISSI, 1998.

Miura, A. and Pritchett, P. L.: Nonlocal stability analysis of the MHD Kelvin-Helmholtz instability in a compressible plasma, J. Geophys. Res., 87, 7431, 1982.

Pedersen, A., et al.: The Wave Experiment Consortium (WEC), Space Science Reviews, 79, 1/2, 93-106, 1997.

Perraut, S., Gendrin, R., Robert, P., and Roux, A.: Magnetic pulsations observed onboard GEOS 2 in the ULF range during multiple magnetopause crossings, in: Proceed. of Magnetospheric Boundary Layer Conference, Alpbach, June 1979, ESA/SP-148, 113-122, 1979.

Pinçon, J. L. and Motschmann, U.: Multi-Spacecraft Filtering: general framework, in: Analysis Methods for multi-spacecraft data, (Eds) Paschmann, G. and Daly, P. W., ISSI, 1998.

Rème, H., et al.: The Cluster Ion Spectrometry (CIS) Experiment, Space Science Reviews, 79 1/2, 303-350, 1997.

Rezeau, L., Morane, A., Perraut, S., Roux, A., and Schmidt, R.: Characterization of Alfvénic fluctuations in the magnetopause boundary layer, J. Geophys. Res., 94, 101-110, 1989.

Rezeau, L., Roux, A., and Russell, C. T.: Can ULF fluctuations observed at the magnetopause play a role in anomalous diffusion?, in: Proceedings of the 26th ESLAB Symposium on 'Study of the Solar- Terrestrial System', ESA SP-346, 127-131, 1992.

Rezeau, L., Belmont, G., Briand, C., Cornilleau-Wehrlin, N., and Reberac, F.: Spectral law and polarization properties of the low frequency waves at the magnetopause, Geophys. Res. Lett., 26, 651-654, 1999.

Safrankova, J., Nemecek, Z., Prech, L., Zastenker, G., Fedorov, A., Romanov, S., Sibeck, D., and Simunek, J.: Two point observation of the magnetopause motion: the Interball project, Adv. Space Res., 20, 801-807, 1997.

Sibeck, D. G., Lopez, R. E., and Roelof, E. C.: Solar wind control of the magnetopause shape, location and motion, J. Geophys. Res., 96, 5489-5495, 1991.

Sonnerüp, B. U. Ö. and Scheible, M.: Minimum and maximum variance analysis, in: Analysis Methods for multi-spacecraft data, (Eds) Paschmann, G. and Daly, P. W., ISSI, 1998. 